\title{
Electron Capture Rates for Neutron Star Crusts
}

\author{
Ana D. Becerril Reyes, Sanjib Gupta, and Hendrik Schatz \\ National Superconducting Cyclotron Laboratory, Joint Institute for Nuclear Astrophysics, and \\ Department of Physics and Astronomy, \\ Michigan State University, East Lansing, MI 48824. \\ E-mail: becerrilenscl.msu.edu, guptasanjibelanl.gov \\ schatzenscl.msu.edu
}

\section{Karl-Ludwig Kratz}

MPI für Chemie, Otto Hahn Institut, Joh. J. Becherweg 27, and HGF VISTARS, D-55128 Mainz, Germany.

E-mail: klkratz@uni-mainz.de

\section{Peter Möller}

Theoretical Division, Los Alamos National Laboratory, NM 87545.

E-mail: mollerelanl.gov

In the study of the crust of an accreting neutron star, electron capture rates on nuclei in the mass range $A=20-106$ from the neutron deficient region to the neutron drip line are needed. At the low temperatures typical of a neutron star crust $\left(T_{9} \sim 0.4\right)$ the determination of the phase space poses a problem since the integrand is very sharply peaked and an error in finding the peak using numerical differentiation or cruder maximum-locator methods will result in inaccuracies at the energies where the integrand has its highest value. In this work we present a global set of temperature and density-dependent continuum electron capture rates and a fast phase space calculator valid for low temperatures. The electron capture rates have been calculated using Gamow-Teller strength distributions from the quasi-particle random-phase approximation (QRPA, [1]) nuclear model. We present a new analytic technique to carry out the evaluation of the phase space for electron capture that is fast enough to be implemented in a reaction network. The integral is split into two separate terms at the chemical potential of the electron gas, thus eliminating the need to find the peak of the integrand. The Coulomb correction, which is essentially constant over the range of energies considered, is taken outside the integral and is evaluated at a suitable effective energy which is a function of the charge of the capturing nucleus, the temperature, the chemical potential and the threshold. We compare our calculated electron capture rates to the compilations by Fuller, Fowler and Newman [2] and by Langanke and Martínez-Pinedo [3].

International Symposium on Nuclear Astrophysics - Nuclei in the Cosmos - IX

25-30 June 2006

CERN

\footnotetext{
${ }^{*}$ Currently at Theoretical Division, Los Alamos National Laboratory, NM 87545.
} 


\section{Motivation}

Observed X-ray bursts in the universe have been explained as the result of thermonuclear explosions on the surface of a neutron star accreting matter from a companion in a binary system. Superbursts are extremely powerful X-ray bursts in which the energy release is $\sim 10^{3}$ times that of a regular burst. They occur with less frequency but have longer cooling timescales (on the order of hours). Superbursts show the same spectral behavior as a normal X-ray burst and hence they are thought to have a thermonuclear nature too, being fueled by the ashes of regular X-ray bursts. Thus, a detailed understanding of the underlying nuclear physics is needed in the study of these phenomena [ []. Deep crust processes, like electron captures and pycnonuclear fusion are crucial in these studies since they determine the composition and thermal properties of the neutron star crust.

At accretion rates of $\sim 10^{-8}-10^{-10} M_{\odot} y r^{-1}$, a neutron star can accrete enough material from its companion to replace its entire crust with ashes of $\mathrm{H}$ and $\mathrm{He}$ burning not in nuclear statistical equilibrium (NSE). The electron chemical potential rises with density as the ashes are pushed deeper in the crust, switching on energetically unfavorable electron capture reactions, which beyond the neutron drip line are accompanied by neutron emission. These reactions produce very neutron-rich unstable nuclei and their energy generation contributes to the heating of the crust. This energy release is one of the observables in transient X-ray binary systems [ $₫$ ]. The neutron star crust composition and its thermal state also affects the crust electrical conductivity. This is important for another fundamental open question -the existence of two classes of X-ray binaries: $\mathrm{X}$-ray pulsars with high magnetic fi elds and X-ray bursters with low magnetic fi elds.

To accurately describe the nuclear processes that take place in the crust it is necessary to start with realistic ashes predicted by a Type-I X-ray burst calculation. For this, electron capture rates on nuclei in the mass range $A=20$ to 106 from the neutron defi cient side to the neutron dripline are needed. Therefore, a global set of electron capture rates is of great importance and it could also serve as input for simulations on massive star evolution or core-collapse supernovae.

Another issue faced in neutron star crust simulations is the calculation of the electron capture phase space. At low temperatures the threshold effects of the increasing density cannot be accurately determined from tables. On the other hand, numerical integration schemes are too timeconsuming for real-time implementation in a reaction network. The exact density at which an electron capture is triggered depends sensitively on the nuclear mass difference between parent and daughter nuclei (ground-state threshold) as well as on the energy of the state in the daughter to which the capture occurs. Thus, interpolation on a coarse density grid is likely to yield highly inaccurate results.

Therefore, a precise neutron star crust simulation requires, in addition to a global set of electron capture rates, an accurate and fast phase space approximation. We present here solutions to both problems: we have developed an analytic technique to accurately compute the low-temperature reaction phase space at high density, especially when the electron chemical potential $\mu_{e}$ is just below the threshold for electron capture (referred to as "pre-threshold" capture). The details of this phase space approximation are described in Section 2 . Also, we have compiled a global set of electron capture rates, which are described in Section 7 with comparisons to rates published by Fuller, Fowler and Newman (henceforth FFN, [2]) and by Langanke and Martínez-Pinedo (hencefort LMP, [3]) for smaller subsets of nuclei. 


\section{Electron capture phase space}

The electron capture rate for a channel designated " $i j^{\prime \prime}\left(i^{t h}\right.$ state of parent/capturing nucleus, $j^{\text {th }}$ state of daughter nucleus)

$$
R=\frac{\ln (2) F_{i j}\left(T_{9}, \mu_{e}, w, q\right)}{f t_{1 / 2}(I \rightarrow J)}
$$

is traditionally evaluated by a numerical integration to obtain the phase space $F_{i j}$ available for the transition

$$
F_{i j}=\int_{w_{l}}^{\infty} w^{2}(q+w)^{2} G(Z, w)\left\{S_{-}\left(w, \mu_{e}\right)\left(1-S_{v}\right)\right\} d w,
$$

where the electron total energy, $w$, is in electron rest mass units $\left(m_{e} c^{2}\right)$ and $q$ is the nuclear mass difference $q_{g s}$ between parent and daughter ground states in $\left(m_{e} c^{2}\right)$ units corrected by the excitation energies of the initial and fi nal states to yield the channel $Q$-value: $q=\left\{q_{g s}+\left(E_{i}-E_{f}\right)\right\} / m_{e} c^{2}$. The capture threshold, $w_{l}$, is unity (zero momentum) if $q \geqslant-1$ and $|q|$ otherwise. We can calculate the temperature and density-dependent state-to-state rate given matrix element information for the transition in the form of a $\log f t$-value: $\lambda_{i j}=\frac{(\ln 2) F_{i j}}{\left(f t_{1 / 2}\right)_{i j}} \simeq \frac{0.693 F_{i j}}{\left(f t_{1 / 2}\right)_{i j}}$ and a total capture rate on the parent nucleus by summing the total destruction rate of all states $j$ in the daughter.

\section{Terms in the integrand}

The distortion of the electronic wavefunction near the capturing nucleus is given by $G(Z, w)=$ $(p / w) F(Z, w)$, a slowly varying function of electron total energy that allows the integrand to be written without explicit reference to the electron momentum $p=\left(w^{2}-1\right)^{\frac{1}{2}}$. Our technique factors out $G(Z, w)$, but not as a constant. Rather, we represent the effect of the Coulomb correction as a temperature-dependent multiplier, whose value reflects the portion of the electron energy spectrum that is actually of importance in the integration at that temperature and density. Hence, we may rewrite the phase-space integral as $F_{i j}=\left\langle G\left(T_{9}, \rho Y_{e}\right)\right\rangle \int_{w_{l}}^{\infty} w^{2}(q+w)^{2}\left\{S_{-}\left(w, \mu_{e}\right)\left(1-S_{v}\right)\right\} d w=$ $\langle G\rangle \int_{w_{l}}^{\infty} P(w) S\left(w, \mu_{e}\right) d w$, with the remaining integration now represented as a convolution of the polynomial $P(q, w)=P(w)=w^{4}+2 q w^{3}+q^{2} w^{2}$ in the energy $w$ with the product of lepton distribution functions. We also assume that neutrino blocking is negligible $\left(S_{v}=0\right)$. We write $S_{-}\left(w, \mu_{e}\right) \equiv S\left(w, \mu_{e}\right)$ to simplify the notation since only electron captures will be discussed here. Without neutrino blocking the fi lter is simply the Fermi-Dirac distribution for relativistic degenerate electrons $S\left(w, \mu_{e}\right)=\left\{1+e^{\left(w-\mu_{e}\right) / k T}\right\}^{-1}=\left\{1+e^{a\left(w-\mu_{e}\right)}\right\}^{-1}$ where the electron total chemical potential (including rest mass) sets the filter bandwidth at a given temperature and density. All energies being in units of $\left(m_{e} c^{2}\right)$, and temperatures being in units of $10^{9} \mathrm{~K}$, we subsume the Boltzmann constant $k$ in $a=\left(5.93 / T_{9}\right)$. Now this filter may be conveniently expanded in a sum of exponentials. For $w<\mu_{e}$ we can write

$$
\left(1-S^{<}\right)=1+\sum_{n=1}^{\infty}(-1)^{n} e^{n a\left(w-\mu_{e}\right)}=1-\sum_{n=1}^{\infty}(-1)^{n-1} e^{-n a \mu_{e}} e^{n a w}
$$

while for $w>\mu_{e}$ we have 


$$
S^{>}=\left[e^{a\left(w-\mu_{e}\right)}\left\{1+e^{-a\left(w-\mu_{e}\right)}\right\}\right]^{-1}=\sum_{n=1}^{\infty}\left\{(-1)^{n-1} e^{n a \mu_{e}}\right\} e^{-n a w} .
$$

\section{Splitting the integration at the chemical potential}

We notice that the phase space integral has its most interesting behavior in the neighborhood of the chemical potential and thus split the integral into two parts: $f_{i j}^{<}=\langle G\rangle \int_{w_{l}}^{\mu_{e}} P(q, w)\left(1-S^{<}\right) d w$ and $f_{i j}^{>}=\langle G\rangle \int_{\mu_{e}}^{\infty} P(q, w) S^{>} d w$. The first integral tracks the slow leakage of phase space out of the region $w<\mu_{e}$ while the second tracks the effect on the rate of the leakage into $w>\mu_{e}$. The separation of these effects should show us the effect of rising temperature on phase space at constant density. Quadrature schemes that split the integration at the chemical potential and fail to locate the integrand peak correctly risk orders-of-magnitude inaccuracy in the rates. The reason why we wish to reduce the integrand to a product of a polynomial with a sum of exponentials is that this will allow us to make use of the indefi nite integral $\int P_{m}(w) e^{b w} d w=e^{b w} \sum_{k=0}^{k=m}\left\{\frac{(-1)^{k}}{b^{k+1}}\right\} P^{(k)}(w)$ where $P^{(k)}(x)$ is the $\mathrm{k}^{\text {th }}$ derivative of the polynomial $P_{m}(x)$ of degree $m$. Thus the integral is reduced to an evaluation of a rapidly convergent series. The analytic expressions for the phase-space factor that we provide are very easy to implement and computationally faster than numerical integration. We have already implemented them in a realistic model of a Neutron Star Crust employing a full reaction network [8]. The technical difficulties and latent inaccuracies that arise from not locating the peak are bypassed entirely since the divider, the chemical potential, is a quantity that is explicitly computed from temperature and density. Notice that we will be interested in integrals involving $e^{b w}=e^{ \pm \text {naw }}$ and hence will end up with powers of $\left(\frac{1}{b}\right)= \pm\left(\frac{1}{n a}\right)= \pm\left(\frac{T_{9}}{5.93 n}\right)$. The density dependence enters in the form of the $e^{ \pm n a \mu_{e}}$ coeffi cients which are just integral powers of the fugacity $e^{a \mu_{e}}$. With the appropriate coeffi cients of the powers of $w$ in $P(w)$ we get $\left(\int\left(w^{4}+2 q w^{3}+q^{2} w^{2}\right) e^{b w} d w\right) / e^{b w}=$ $\sum_{k=0}^{k=4}(-1)^{k}\left(\frac{1}{b}\right)^{(k+1)} P^{(k)}(w)$ where $P^{(k)}(w)$ is the $k$-th derivative of $P(w)$ with respect to $w$. We now rewrite the phase space integral using the series expansions of the convolving Fermi-Dirac fi lter on either side of the chemical potential to obtain the phase space in terms of three integrals:

$$
\frac{F_{i j}}{<G>}=\left[\frac{\left(f_{i j}^{<}+f_{i j}^{>}\right)}{<G>}\right]=\int_{w_{l}}^{\mu_{e}} P(w) d w-\int_{w_{l}}^{\mu_{e}} P(w) S^{<}\left(w, \mu_{e}\right) d w+\int_{\mu_{e}}^{\infty} P(w) S^{>}\left(w, \mu_{e}\right) d w .
$$

Now, the fir rst integral is simply $\int_{w_{l}}^{\mu_{e}}\left(w^{4}+2 q w^{3}+q^{2} w^{2}\right) d w=\left[\frac{w^{5}}{5}+\frac{2 q w^{4}}{4}+\frac{q^{2} w^{3}}{3}\right]_{w_{l}}^{\mu_{e}}=P_{b}\left(\mu_{e}, w_{l}, q\right)$ and is the analog of the approximately $w_{0}^{5}$ dependence of emission integral phase-space. The steep fall-off of the convolving fi lter beyond the chemical potential renders it the effective "end-point" in the first integral. The second integral measures the "leaking out" of the Fermi-Dirac for $w<\mu_{e}$ with rising temperature,

$$
\begin{aligned}
\int_{w_{l}}^{\mu_{e}} P(w) S^{<}\left(w, \mu_{e}\right) d w & =\int_{w_{l}}^{\mu_{e}}\left\{\sum_{n=1}^{\infty}(-1)^{n-1} e^{-n a \mu_{e}} e^{n a w} P(w)\right\} d w \\
& =\sum_{n=1}^{\infty}(-1)^{n-1} e^{-n a \mu_{e}} \int_{w_{l}}^{\mu_{e}} e^{n a w} P(w) d w
\end{aligned}
$$


Let $z=e^{a\left(w_{l}-\mu_{e}\right)}$ for notational ease. Now, the Dirichlet $\eta$-function is related to the Riemann $\zeta$-function by $\eta(k)=\sum_{n=1}^{\infty} \frac{(-1)^{n-1}}{n^{k}}=\left(1-2^{1-k}\right) \zeta(k)$ for $k \neq 1$. It can be evaluated at unity from the MacLaurin series for $\ln (1+x)$ and shown to be $\eta(1)=\ln 2$. We will also fi nd useful the polylogarithm (generalized logarithm) function $f_{k}(z)=\sum_{n=1}^{\infty} \frac{(-1)^{n-1} z^{n}}{n^{k}}$. Then we can can write the second integral in Eq.(4.1) as

$$
\begin{aligned}
& P_{\text {decr }}\left(\mu_{e}, q\right)=\int_{w_{l}}^{\mu_{e}} P(w) S^{<}\left(w, \mu_{e}\right) d w \\
= & \sum_{k=1}^{k=5}(-1)^{(k-1)} c_{k} P^{(k-1)}\left(\mu_{e}\right) T_{9}^{k}-\sum_{k=1}^{k=5}(-1)^{(k-1)} P^{(k-1)}\left(w_{l}\right)(5.93)^{-k} T_{9}^{k} f_{k}(z)
\end{aligned}
$$

The last integral in Eq. (4.1) is evaluated similarly but with $b=-n a$

$$
\begin{aligned}
& P_{\text {incr }}\left(\mu_{e}, q\right)=\int_{\mu_{e}}^{\infty} P(w) S^{>}\left(w, \mu_{e}\right) d w \\
= & \sum_{n=1}^{\infty}(-1)^{n-1} e^{n a \mu_{e}} \int_{\mu_{e}}^{\infty} e^{-n a w} P(w) d w=\sum_{k=1}^{k=5} c_{k} P^{(k-1)}\left(\mu_{e}\right) T_{9}^{k}
\end{aligned}
$$

where $c_{k}=\eta(k)(5.93)^{-k}$, and $\eta$ is the Dirichlet function. As a result, the total phase space for a specifi ed channel $Q$-value at a chemical potential $\mu_{e}$ and temperature $T_{9}$ is given by

$$
F_{i j}=\left\langle G\left(T_{9}, \mu_{e}\right)\right\rangle f_{i j}=\left\langle G\left(T_{9}, \mu_{e}\right)\right\rangle\left[P_{b}\left(\mu_{e}, w_{l}, q\right)-P_{\mathrm{decr}}\left(\mu_{e}, q\right)+P_{\mathrm{incr}}\left(\mu_{e}, q\right)\right]
$$

\section{Calculations for threshold higher than chemical potential $\left(w_{l}>\mu_{e}\right)$}

For large negative values of channel $Q$-value and consequently very high capture thresholds, $w_{l}=-q>\mu_{e}$, the energy is always higher than the chemical potential, so $S^{>}$can be used throughout the range of integration.

$$
\begin{aligned}
& \int_{w_{l}}^{\infty} \frac{P(w, q)}{1+e^{\left(w-\mu_{e}\right) / k T}} d w=\int_{w_{l}}^{\infty} P(w, q)\left\{\sum_{n=1}^{\infty}(-1)^{n} e^{n a \mu_{e}} e^{-n a w}\right\} d w \\
= & \sum_{n=1}^{\infty}(-1)^{n-1} e^{n a\left(\mu_{e}-w\right)} \sum_{k=1}^{k=5}(n a)^{-k} P^{(k-1)}(w)
\end{aligned}
$$

This expression vanishes at the upper limit to give

$$
\int_{w_{l}}^{\infty} \frac{P(w, q)}{1+e^{\left(w-\mu_{e}\right) / k T}} d w=\sum_{n=1}^{\infty}(-1)^{n-1} e^{n a\left(\mu_{e}-w_{l}\right)} \sum_{k=1}^{k=5}(n a)^{-k} P^{(k-1)}\left(w_{l}\right)
$$

which can be simplifi ed for $m=-q$ to give 


$$
\begin{aligned}
& \sum_{n=1}^{\infty}(-1)^{n-1} e^{n a\left(\mu_{e}-w_{l}\right)}\left\{\left(\frac{1}{n a}\right)^{3}\left(2 w_{l}^{2}\right)+\left(\frac{1}{n a}\right)^{4}\left(12 w_{l}\right)+\left(\frac{1}{n a}\right)^{5}(24)\right\} \\
= & \frac{2 w_{l}^{2} T_{9}^{3}}{(5.93)^{3}} \sum_{n=1}^{\infty} \frac{(-1)^{n-1} z^{n}}{n^{3}}+\frac{12 w_{l} T_{9}^{4}}{(5.93)^{4}} \sum_{n=1}^{\infty} \frac{(-1)^{n-1} z^{n}}{n^{4}}+\frac{24 T_{9}^{5}}{(5.93)^{5}} \sum_{n=1}^{\infty} \frac{(-1)^{n-1} z^{n}}{n^{5}} \\
= & \frac{2 w_{l}^{2} T_{9}^{3}}{(5.93)^{3}} f_{3}(z)+\frac{12 w_{l} T_{9}^{4}}{(5.93)^{4}} f_{4}(z)+\frac{24 T_{9}^{5}}{(5.93)^{5}} f_{5}(z)
\end{aligned}
$$

where $z=e^{a\left(\mu_{e}-w_{l}\right)}=e^{5.93\left(\mu_{e}-w_{l}\right) / T_{9}}$. However for the important special case when $w_{l}=1 \neq$ $-q$ and $w_{l}>\mu_{e}$, the full expression of Eq. (5.3) must be retained and we may also use $f_{n}(z) \approx z$ (when $z<1$, a very useful approximation for further speeding up calculations in a reaction network). Such will be the case at very low electron densities where the total chemical potential is less than the rest mass but $q>-1$. For this situation we can write down the phase space as

$$
\int_{w_{l}}^{\infty} \frac{P(w, q)}{1+e^{\left(w-\mu_{e}\right) / k T}} d w=\sum_{k=1}^{k=5} P^{(k-1)}\left(w_{l}\right) f_{k}(z) T_{9}^{k}
$$

\section{Extraction of the Coulomb correction}

The Coulomb correction $G(Z, w)$ is almost constant over the range of integration for a fi xed temperature and density. One computational treatment would be to fi t a low-order polynomial to it over the energy range and obtain a new polynomial of slightly higher order for the integrand. This may involve more computational effort than necessary for astrophysical purposes. An alternative approach is to assume that $G(Z, w)$ is constant for a fi xed temperature and density, factor it out of the integration and estimate it at a suitable effective energy. Now the reduced phase space (without Coulomb correction) can be used to fi nd the mean of $P(w)=w^{2}(w+q)^{2}$ over the range of integration weighted by the FD distribution $\langle P(w)\rangle=\left(\int_{w_{l}}^{\infty} \frac{P(w, q) d w}{1+e^{\left(w-\mu_{e}\right) / k T}}\right)\left(\int_{w_{l}}^{\infty} \frac{d w}{1+e^{\left(w-\mu_{e}\right) / k T}}\right)^{-1}$ and the denominator can be expressed as $\frac{T_{9} \ln \left(1+e^{c}\right)}{5.93}$ where $c=\left(\frac{\mu_{e}-w_{l}}{k T}\right)=\left(\frac{5.93\left(\mu_{e}-w_{l}\right)}{T_{9}}\right)$. Then we extract the effective energy $w_{\text {eff }}$ using $\langle P(w)\rangle=w_{\text {eff }}^{2}\left(w_{\text {eff }}+q\right)^{2}$ and evaluate the Coulomb correction at this value (see FFN [2] for details of computation using the complex Gamma function).

\section{Comparison of EC rates from QRPA to FFN and LMP.}

Weak-interaction tables for a global set of nuclei without restrictions in model space will be published shortly [9], which we have calculated using Gamow-Teller strength distributions from the QRPA nuclear model.

Here we present a comparison of our calculated electron capture rates to the ones published by Fuller, Fowler \& Newman [2] and to the ones by Langanke \& Martínez-Pinedo [3]. We make these comparisons at a temperature typical of a neutron star crust, $T_{9}=0.4$, and at four different densities: $\log \left(\rho Y_{e}\right)=1.0, \log \left(\rho Y_{e}\right)=8.0, \log \left(\rho Y_{e}\right)=9.0$ and $\log \left(\rho Y_{e}\right)=10.0$. Figure 1 shows the results of the comparison of our calculated electron capture rates to those by FFN. Similarly, Figure 2 shows the results of the comparison of our calculated electron capture rates to those by Langanke 
and Martínez-Pinedo [3]. The color coding gives the log (base 10) of the ratio of rates according to the color bar shown. The ranges of nuclei for which the electron capture rates are compared are determined by two factors: fi rst, the ranges of nuclei for which FFN and LMP (respectively) provide calculated rates, and second, the lower limit of $10^{-17} s^{-1}$ imposed to all the rates entering the comparisons.

As the density rises it becomes possible to access the low-lying states of increasingly neutronrich nuclei. Thus, the quasiparticle energies in the QRPA, when compared to the level assignments used in FFN or LMP, result in different low-lying strengths in the nuclei accessed by the rising $\mu_{e}$. As can be seen in both Fig. 1 and Fig. 2, the effects of deformation show up very clearly. Significantly deformed neutron orbits in neutron-rich nuclei can increase the number of daughter states accessible from the $N=4$ oscillator shell due to the electron chemical potential and increase the rates even at low temperatures. This effect is of great signifi cance for reaction networks employed in supernova simulations since it alerts us of important limitations of interacting spherical shell model approaches - realistic residual interactions will mix the $p f$ shell with the sdg shell resulting in confi gurations accessible at stellar temperatures. Moreover, this effect is magnifi ed by the low- $\mathrm{j}$ orbitals from e.g. the $g_{9 / 2}$ shell due to the explicit inclusion of deformation in our model. As a further example, as Radioactive Ion-Beam (RIB) facilities probe increasingly exotic nuclei important to r-process nucleosynthesis up to the $\mathrm{N}=82$ shell closure and beyond, the deformed proton $g_{7 / 2}$ and neutron $d_{5 / 2}, g_{7 / 2}$ and $h_{11 / 2}$ orbits will prove to be crucial determinants of nuclear structure.

There is good agreement with FFN and LMP rates for proton-rich nuclei because we do not need to go beyond the $p f$-shell in protons for the nuclei under consideration. However, the effects of the rising chemical potential show the very different effects of thresholds becoming accessible on increasingly neutron-rich nuclei with negative $Q$-values. Here we specifi cally list some $\mathrm{Fe}$ group isotopes for which our rates differ by more than an order of magnitude from FFN and LMP. In our comparison with the spherical independent-particle model of FFN we fi nd at $T_{9}=0.4$ and $\rho Y_{e}=10^{9} \mathrm{~g} / \mathrm{cc}$ (corresponding to a driving force of $\mu_{e}=5.182 \mathrm{MeV}$ - a very important region of heat deposition from electron capture in the crust since it is so close to the site of carbon ignition) ${ }^{48,51} \mathrm{~V},{ }^{50,52} \mathrm{Cr},{ }^{54,58,60} \mathrm{Mn},{ }^{55,59} \mathrm{Fe},{ }^{55,58,60} \mathrm{Co}$, and ${ }^{58} \mathrm{Ni}$. The comparison with LMP rates results in the isotope list ${ }^{49,51,54} \mathrm{~V},{ }^{52} \mathrm{Cr},{ }^{53,57,58} \mathrm{Mn},{ }^{55,59} \mathrm{Fe},{ }^{59,60,61,62,64} \mathrm{Co}$, and ${ }^{60,61,63} \mathrm{Ni}$. A detailed study of nuclear structure differences between our model and the FFN and LMP ones, as well as comparisons with charge-exchange experiment information is beyond the scope of this work but is currently being undertaken and will be published soon [10].

\section{Conclusion}

We have presented a global set of electron capture rates and a fast phase space calculator that is valid for low temperatures. Therefore, now the input and mathematical tools for neutron star crust calculations are avaliable. For the fi rst application and the important impact of these rates on a neutron star crust reaction network see E. Brown et al. in these proceedings [7]. A promising further enhancement will be to add excited states in the parent nuclei, so that the compilation in [9] will evolve and provide a global set of electron capture rates applicable to a wide range of astrophysical phenomena including supernovae. 
Acknowledgements

This work is supported by the Joint Institute for Nuclear Astrophysics under NSF-PFC grant PHY 02-16783 and by NSF grant PHY 0110253. KLK acknowledges support from Virtuelles Institut für Struktur der Kerne und Nukleare Astrophysik (VISTARS) under HGF grant VH-VI-061. This work was partially carried out under the auspices of the National Nuclear Security Administration of the U.S. Department of Energy at Los Alamos National Laboratory under Contract No. DE-AC52-06NA25396.

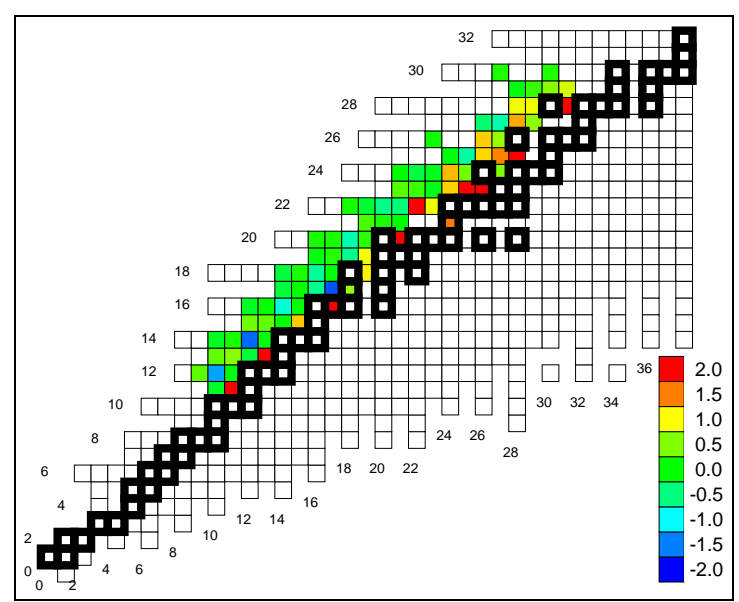

(a) $\log \left(\rho Y_{e}\right)=1.0, \mu_{e}\left(T_{9}=0.4\right)=0.205 \mathrm{MeV}$

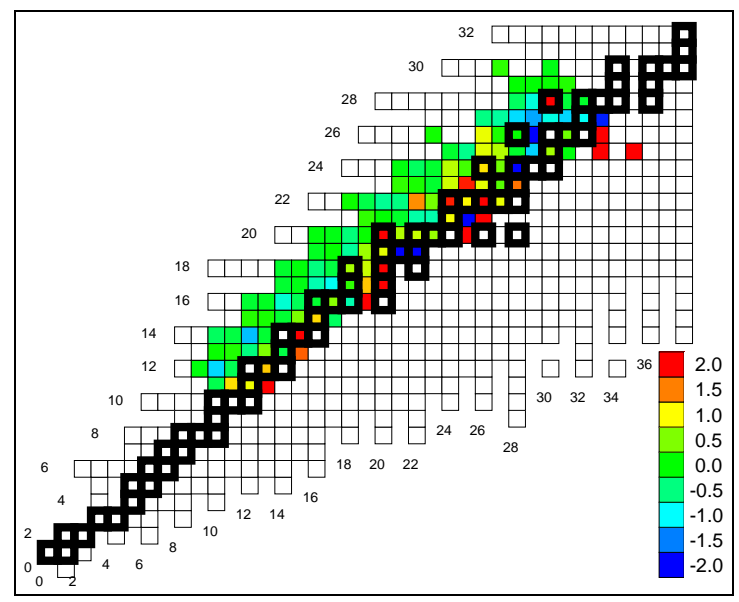

(c) $\log \left(\rho Y_{e}\right)=9.0, \mu_{e}\left(T_{9}=0.4\right)=5.182 \mathrm{MeV}$

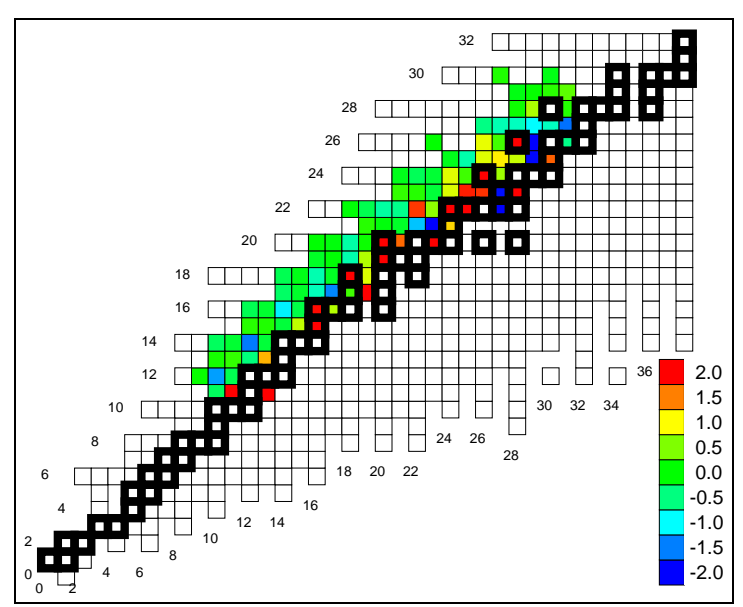

(b) $\log \left(\rho Y_{e}\right)=8.0, \mu_{e}\left(T_{9}=0.4\right)=2.445 \mathrm{MeV}$

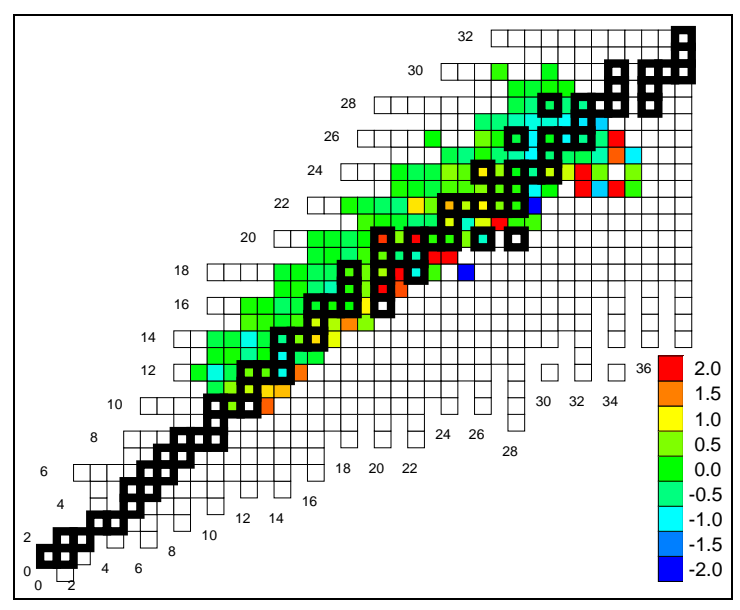

(d) $\log \left(\rho Y_{e}\right)=10.0, \mu_{e}\left(T_{9}=0.4\right)=11.119 \mathrm{MeV}$

Figure 1: Comparison of our calculated ec-rates to those calculated by FFN. The color coding gives the Log of the ratio (our-rates/FFN-rates) according to the color bar at the bottom. Thick black borders denote stable nuclei. 


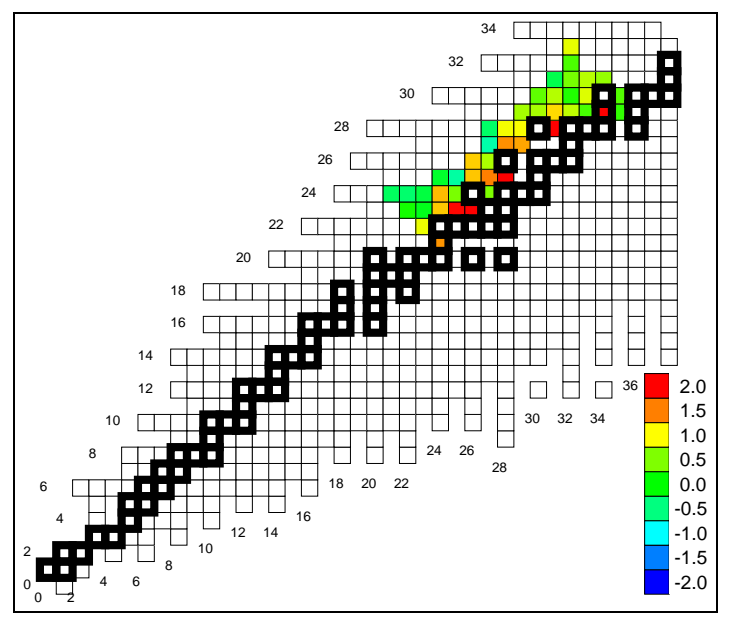

(a) $\log \left(\rho Y_{e}\right)=1.0, \mu_{e}\left(T_{9}=0.4\right)=0.205 \mathrm{MeV}$

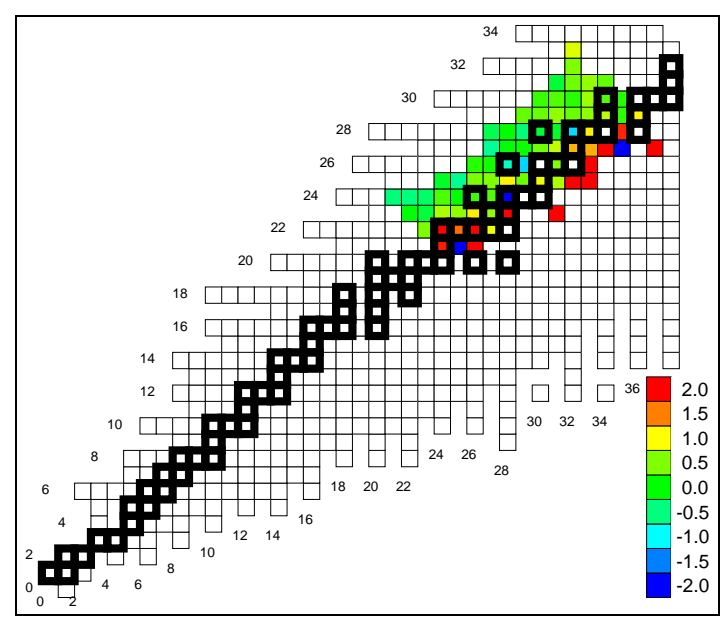

(c) $\log \left(\rho Y_{e}\right)=9.0, \mu_{e}\left(T_{9}=0.4\right)=5.182 \mathrm{MeV}$

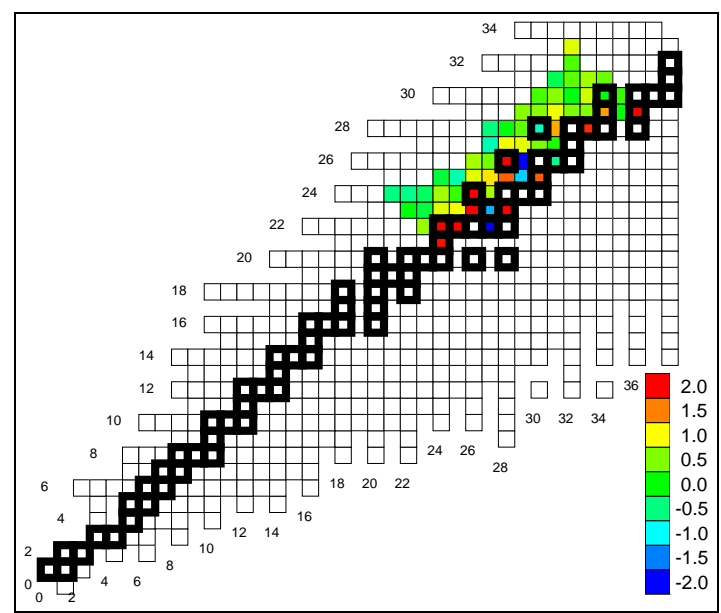

(b) $\log \left(\rho Y_{e}\right)=8.0, \mu_{e}\left(T_{9}=0.4\right)=2.445 \mathrm{MeV}$

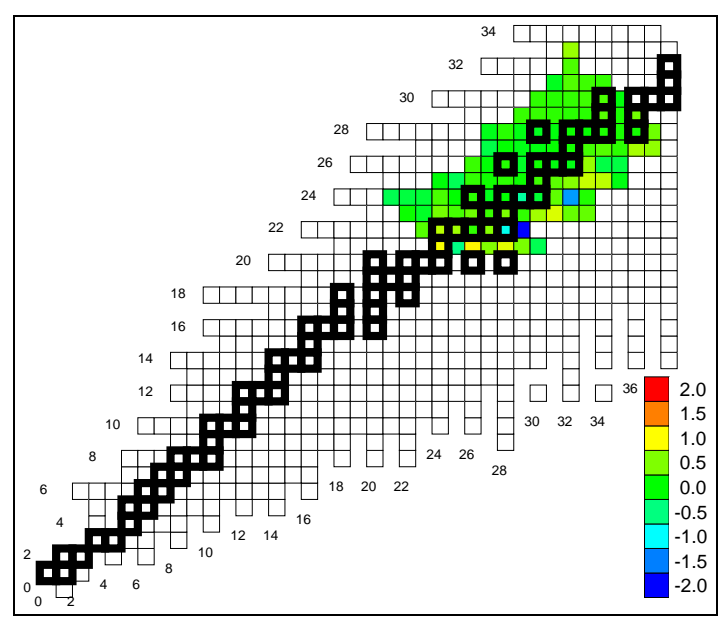

(d) $\log \left(\rho Y_{e}\right)=10.0, \mu_{e}\left(T_{9}=0.4\right)=11.119 \mathrm{MeV}$

Figure 2: Comparison of our calculated ec-rates to those by LMP. The color coding gives the log of the ratio (our-rates/LMP-rates) according to the color bar at the bottom. Thick black borders denote stable nuclei.

\section{References}

[1] P. Möller \& J. Randrup, Nuc. Phys. A. 514, 1-48 (1990).

[2] G. Fuller, W. Fowler \& M. Newman, Ap. J. Suppl. Ser. 42, 447-473 (1980).

[3] K. Langanke \& G. Martínez-Pinedo, At. Data and Nuc. Data Tables. 79, 1-346 (2001).

[4] H. Schatz \& K.E. Rehm., Nuc. Phys. A. 777 601-622 (2006).

[5] P. Haensel \& J.L. Zdunik, Astron. Astrophys. 227, 431-436 (1990).

[6] P. Haensel \& J.L. Zdunik, Astron. Astrophys. 404, L33-L36 (2003). 
[7] E. Brown, S. Gupta, H. Schatz, P. Möller \& K.L. Kratz. Electron capture reactions in neutron star crusts: deep heating and observational constraints. In Proceedings of NIC-IX Conference. PoS(NIC-IX)046

[8] S. Gupta, E. Brown, H. Schatz, P. Möller \& K.L. Kratz. Submitted to Ap. J. (2006). [astro-ph/0609828].

[9] S. Gupta, P. Möller, K.L. Kratz \& H. Schatz . In preparation.

[10] S. Gupta, K.L. Kratz \& P. Möller. In preparation. 